\title{
Numerical Fitting of Molecular Properties to Hermite Gaussians
}

\author{
G. Andrés Cisneros, ${ }^{*} \dagger$ Dennis Elking, ${ }^{\dagger}$ Jean-Philip Piquemal, $\stackrel{\ddagger}{\ddagger}$ and Thomas A. Darden ${ }^{\dagger}$ \\ Laboratory of Structural Biology, National Institute of Environmental Health Sciences, Research Triangle Park \\ (RTP), North Carolina 27709, and Laboratoire de Chimie Théorique, UMR 7616 CNRS, Université \\ Pierre-et-Marie-Curie, case courrier 137, 4, place Jussieu, 75252 Paris, France
}

Received: June 20, 2007; In Final Form: August 9, 2007

\begin{abstract}
A procedure is presented to fit gridded molecular properties to auxiliary basis sets (ABSs) of Hermite Gaussians, analogous to the density fitting (DF) method (Dunlap; et al. J. Chem. Phys. 1979, 71, 4993). In this procedure, the $a b$ initio calculated properties (density, electrostatic potential, and/or electric field) are fitted via a linearor nonlinear-least-squares procedure to auxiliary basis sets (ABS). The calculated fitting coefficients from the numerical grids are shown to be more robust than analytic density fitting due to the neglect of the core contributions. The fitting coefficients are tested by calculating intermolecular Coulomb and exchange interactions for a set of dimers. It is shown that the numerical instabilities observed in DF are caused by the attempt of the ABS to fit the core contributions. In addition, this new approach allows us to reduce the number of functions required to obtain an accurate fit. This results in decreased computational cost, which is shown by calculating the Coulomb energy of a 4096 water box in periodic boundary conditions. Using atom centered Hermite Gaussians, this calculation is only 1 order of magnitude slower than conventional atomcentered point charges.
\end{abstract}

\section{Introduction}

The simulation of molecular systems is a field of intense study. Ideally, calculations should be performed with quantum mechanical methods, however, approximations have to be done to reduce computational cost. An example is the simulation of biomolecular systems, where empirical force fields are used for the calculation. ${ }^{1-5}$ In general, these force fields rely on the separation of bonded and nonbonded terms to calculate the intraand intermolecular contributions. In the latter case, nonbonded interactions are separated into a Coulomb contribution determined by pairwise interactions of point charges centered on atoms, and a 6-12 Van der Waals term that accounts for the exchange and dispersion contributions. ${ }^{6}$ In most cases atom centered point charges are employed to calculate the Coulomb term. Several methods have been proposed to obtain these charges such as population analysis, ${ }^{7}$ atoms in molecules, ${ }^{8}$ and electrostatic potential (ESP) fitting, ${ }^{9-11}$ to name a few.

Multipolar expansions ${ }^{12-16}$ have been used to improve the Coulomb calculation. ${ }^{17-19}$ Unfortunately, this approach lacks the ability to describe the charge penetration effects observed in continuous charge distributions. Solutions to this problem have been proposed in the form of damping functions. ${ }^{20-22}$

We have shown that the Coulomb interaction can be determined accurately by interacting frozen densities. ${ }^{23}$ The monomer frozen densities are obtained using the density fitting (DF) method. ${ }^{24-26}$ Here the electron density is expanded on auxiliary basis sets (ABSs) centered on specific sites on the molecule. This methodology has been the basis for the Gaussian electrostatic model-0 (GEM-0), where each component of the $a b$ initio energy decomposition (namely, Coulomb, exchangerepulsion, polarization, and charge transfer) is calculated with densities fitted to s-type Gaussian functions. ${ }^{27}$

\footnotetext{
$\dagger$ National Institute of Environmental Health Sciences.

Université Pierre-et-Marie-Curie.
}

GEM-0 has been extended by introducing a fitting basis based on Hermite Gaussians with angular momentum larger than 0 (s-type functions). ${ }^{28}$ The use of Hermite Gaussians leads to a natural multipolar decomposition on the fitting sites, which is intrinsically finite of order equal to the highest angular momentum of the ABS employed in the fit. Unfortunately, the DF procedure employed to determine the fitting coefficients presents some numerical instability (noise) problems. ${ }^{28}$

Recently, we have employed Gaussian multipoles to account for polarization in classical force fields. ${ }^{29}$ There it was shown that the use of continuous functions gives a more accurate interaction than their discrete counterparts. On the basis of these results, we are motivated in this contribution to explore whether the use of Gaussians for the fitting of molecular properties results in a more robust fit, because they allow the exploration of points closer to the core than point multipoles. We present two fitting procedures. The first one relies on linear least squares to fit GEM coefficients for different ABSs to gridded molecular properties. Additionally, we present results for a nonlinear fit to s-type Gaussians where the exponents of the basis and the fitting coefficients are optimized concurrently. The use of gridded molecular properties allows us to avoid fitting points near the core that show up as outliers in the standard least squares fit, in a manner similar to ESP fitting. ${ }^{9-11}$

In the following section we discuss the methodologies employed for the fitting procedure. Subsequently, we present results for the determination of intermolecular electrostatic and exchange interaction energies for a set of molecules. These results are compared to constrained space orbital variations $(\mathrm{CSOV})^{30,31}$ decomposition results.

\section{Methods}

In this section we present the theory and computational details used for this study. In subsection 2.1 we provide a brief overview of the DF method and linear-least-squares and 
nonlinear-least-squares procedures as applied to Hermite Gaussians. Subsequently, in subsection 2.2 we briefly review the methods to calculate intermolecular Coulomb and exchange energies from fitted densities. Finally, in subsection 2.3 we present the details of the calculations.

2.1. Least-Squares Methods. As mentioned above, the intermolecular contribution can be calculated from frozen density obtained from DF methodology. Here, the approximate density can be obtained by fitting the analytic molecular density using an ABS, $\tilde{\rho}=\sum_{k} c_{k} k(r)$, where $\tilde{\rho}$ is the approximate density, $k(r)$ are the Hermite Gaussians of the ABS and $c_{k}$ are the fitting coefficients.

The fitting coefficients are obtained by minimizing the Coulomb self-interaction energy of the error $\left(E_{\text {self }}\right){ }^{25,26}$ This procedure leads to a linear system of equations:

$$
\frac{\partial E_{\text {self }}}{\partial c_{l}}=-\sum_{\mu, v} P_{\mu \nu}\langle u v|| l\rangle+\sum_{k} c_{k}\langle k|| l\rangle
$$

where $P_{\mu \nu}$ is the $a b$ initio density. From eqs 1 above, the coefficients $c_{k}$ can be determined by setting $c=G^{-1} j$, where $j$ $=\sum_{\mu, \nu} P_{\mu \nu}\langle u \nu|| l\rangle$ and $G=\langle k|| l\rangle$. In principle, $G$ is symmetric and positive definite; in practice however, this matrix is almost singular and therefore care must be taken during the diagonalization to obtain its inverse. Previously we have used both singular value decomposition (SVD) and Tikhonov regularization $^{32}$ to diagonalize $G .^{23,28}$ In these studies we observed that noise is still a problem, which was suggested to come from the attempt of the ABSs to fit the density at the nuclear cores.

In the present contribution, we are interested instead in using grids of molecular properties for the fitting procedure instead of the analytic density matrix. The use of numerical grids allows the discarding of points at and near the core, thus effectively neglecting the core contributions. In this case, this is achieved by minimizing the following fitting function:

$$
\chi^{2}=\sum_{\alpha} w_{\alpha} \sum_{i} W\left(r_{i}\right)\left(y_{\alpha}\left(\mathbf{r}_{i}\right)-\tilde{y}_{\alpha}\left(\mathbf{r}_{i}, c_{k}\right)\right)^{2}
$$

where $y_{\alpha}\left(\mathbf{r}_{i}\right)$ corresponds to the ab initio molecular property of interest at point $i$, and $\tilde{y}_{\alpha}\left(\mathbf{r}_{i}, c_{k}\right)$ is the same property evaluated with the $k$ th ABS element at the same point on the grid, and $\alpha$ denotes the molecular property(ies) of interest. For example, if the molecular property of interest is the density, $\rho(r)$, then $y_{\rho}\left(\mathbf{r}_{i}\right)=\rho\left(r_{i}\right)$, and $\tilde{y}_{\rho}\left(\mathbf{r}_{i}, c_{k}\right)=\sum_{k} k_{k}\left(r_{i}\right)$. Finally, $W(r)$ is the weighting function for the point on the grid, which can be defined in several ways, ${ }^{10,11}$ and $w_{\alpha}$ is the relative weight for property $\alpha$.

Equation 2 can be minimized by using the Newton Raphson (NR) method. Suppose $\mathbf{c}^{0}$ is a column vector of an initial guess set of parameters; then a better estimate of the parameters $\mathrm{c}$ can be found by solving

$$
\mathbf{c}-\mathbf{c}^{0}=-\mathbf{H}^{0,-1} \mathbf{g}^{0}
$$

where $\mathbf{g}^{0}$ is the gradient and $\mathbf{H}^{0}$ is the Hessian of $\chi^{2}$ with repsect to $\mathbf{c}$ evaluated at $\mathbf{c}^{0}$. In the case of a linear optimization, $\chi^{2}$ is a quadratic function of the parameters and the NR method is exact, i.e., the optimal set of parameters $\mathbf{c}$ can be obtained by solving eq 3 once.

In the case of nonlinear optimization, that is, when $\tilde{y}_{\alpha}\left(\mathbf{r}_{i}, c_{k}\right)$ is a nonlinear function of the parameters $c_{k}$ (e.g., of the Gaussian exponents), eq 3 has to be solved iteratively. When the parameter guess $\mathbf{c}^{0}$ is near the optimal set of parameters, the Hessian can be approximated by neglecting the second derivative of $\tilde{y}_{\alpha}\left(\mathbf{r}_{i}\right)$.
However, when the parameter guess is far from the minimum, a steepest descent search direction should be used. The Levenberg-Marquardt nonlinear-least-squares fitting algorithm ${ }^{32}$ prescribes adding a large constant matrix to $\mathbf{H}^{0}$ to take small steps in the direction of the gradient, i.e., let $\mathbf{F} \equiv \mathbf{H}^{0}+$ $\omega \mathbf{I}$, and solve $\mathbf{c}-\mathbf{c}^{0}=-\mathbf{F}^{-1} \mathbf{g}^{0}$. As the estimate for the best set of parameters $\mathbf{c}$ iteratively improve, $\omega$ gradually lowers until $\mathbf{F} \simeq \mathbf{H}^{0}$. At this point, the parameters converge quadratically near the minimum.

As was the case for the analytic DF in our previous study, we have used Tikhonov regularization for the inversion of the Hessian for the linear-least-squares procedure. In this method, the equation to be minimized is modified by adding $\lambda \sum_{k} c_{k}$ to eqs 1 or 2 . In this way, the redundant basis set contributions can be penalized by modifying $\lambda .{ }^{28}$ In this case, as in our previous studies, we have used a reference local frame for each fitting site to transfer the calculated fitting coefficients. ${ }^{28}$

2.2. Intermolecular Coulomb and Exchange Interactions. We have previously shown that Coulomb and exchange intermolecular interactions may be calculated from frozen monomer fitted densities. ${ }^{23,27,28}$ In the former case, the Coulomb interaction using fitted densities $\tilde{\rho}$ is obtained by

$$
\begin{aligned}
E_{\text {Coulomb }}= & \sum_{i} \sum_{j} \frac{Z_{\mathrm{A}_{i}} Z_{\mathrm{B}_{j}}}{r_{\mathrm{A}_{i} \mathrm{~B}_{j}}}+\sum_{i} \int \frac{Z_{\mathrm{A}_{i}} \tilde{\rho}^{\mathrm{B}}\left(r_{2}\right)}{r_{\mathrm{A}_{i} 2}} \mathrm{~d} r_{2}+ \\
& \sum_{j} \frac{Z_{\mathrm{B}_{j}} \tilde{\rho}^{\mathrm{A}}\left(r_{1}\right)}{r_{1 \mathrm{~B}_{j}}} \mathrm{~d} r_{1}+\iint \frac{\tilde{\rho}^{\mathrm{A}}\left(r_{1}\right) \tilde{\rho}^{\mathrm{B}}\left(r_{2}\right)}{r_{12}} \mathrm{~d} r_{1} \mathrm{~d} r_{2}
\end{aligned}
$$

where $Z_{\mathrm{A}_{i}}$ represents the nuclei on molecule $\mathrm{A}, \tilde{\rho}^{\mathrm{A}}$ represents the approximate density of molecule $\mathrm{A}, Z_{\mathrm{B}_{j}}$ represents the nuclei on molecule $\mathrm{B}$, and $\tilde{\rho}^{\mathrm{B}}$ represents the approximate density of molecule B.

In the case of the exchange contribution, we have used the overlap model initially proposed by Wheatley and Price, ${ }^{33}$ which we have shown to be applicable using the DF formalism. ${ }^{27}$

$$
E_{\text {exchange }}=K \Omega_{\mathrm{r}}
$$

Here $K$ is a fitting parameter obtained from a linear regression of the overlap of charge density versus the CSOV value, and $\Omega_{\mathrm{r}} \approx \int \tilde{\rho}^{\mathrm{A}}\left(r_{\mathrm{A}}\right) \tilde{\rho}^{\mathrm{B}}\left(r_{\mathrm{B}}\right) \mathrm{d} r$ is the overlap of fitted densities.

2.3. Computational Methods. The above procedures were implemented on a Fortran90 program developed by the authors. In all cases, the grids for the molecular properties were generated from relaxed one-electron density matrices obtained at the B3LYP/6-31G(d) level using the Gaussian98 program. ${ }^{34}$ Once the desired density matrix was obtained, our program was employed to generate the desired grids, which were in turn used to fit the coefficients. In the case of the linear-least-squares method, a grid step-size of $0.17 \AA$ was employed, whereas for the nonlinear fit, the step-size was reduced to $0.05 \AA$. The ABSs employed are A1 and P1 as used in our previous studies unless stated otherwise, also, the geometries of the 10 water dimers correspond to those investigated previously. ${ }^{28}$

We have considered three molecular properties for the linearleast-squares fit: density, ESP and electric field. Additionally, we have investigated the fitting procedure using all these properties weighted evenly. Rectangular grids with evenly spaced points have been implemented in our code to test the fitting procedure.

In all cases the weighing function was used such that points on the grid that were at or below a certain cutoff distance to 
TABLE 1: Absolute Average (Maximum) Error in Coulomb Interaction, in kcal/mol, with Respect to CSOV Using Hermite Gaussians for the 10 Water Dimers When Fitting Grids to A1 on 5 Sites $^{a}$

\begin{tabular}{lcccccc}
\hline & \multicolumn{5}{c}{ cutoff $(\mathrm{au})$} \\
\cline { 2 - 5 } cube(s) & 1.0 & 0.8 & 0.6 & 0.5 & 0.4 & 0.2 \\
\hline all & $0.20(0.51)$ & $0.23(0.47)$ & $0.25(0.48)$ & $0.27(0.48)$ & & \\
density & $1.45(3.06)$ & $1.40(2.53)$ & $1.38(2.45)$ & $1.44(2.46)$ & $1.44(2.46)$ & $0.23(0.50)$ \\
ESP & $0.06(0.16)$ & $0.12(0.28)$ & $0.15(0.39)$ & $0.15(0.39)$ & $0.15(0.39)$ & $0.18(0.39)$ \\
field & $0.26(0.54)$ & $0.14(0.28)$ & $0.18(0.43)$ & $0.21(0.56)$ & $0.21(0.56)$ & $0.22(0.60)$
\end{tabular}

${ }^{a}$ Cutoffs are in au. "All" refers to fitting all cubes with even weight.

TABLE 2: Absolute Average (Maximum) Error in Coulomb Interaction, in kcal/mol, with Respect to CSOV Using Hermite Gaussians for the 10 Water Dimers When Fitting Grids to P1 on 5 Sites $^{a}$

\begin{tabular}{lcccccc}
\hline & \multicolumn{5}{c}{ cutoff $(\mathrm{au})$} \\
\cline { 2 - 5 } cube(s) & 1.0 & 0.8 & 0.6 & 0.5 & 0.4 & 0.2 \\
\hline all & $0.07(0.13)$ & $0.09(0.18)$ & $0.10(0.21)$ & $0.11(0.22)$ & $0.23(0.75)$ & $0.19(0.75)$ \\
density & $2.80(5.93)$ & $1.45(3.07)$ & $0.67(2.27)$ & $0.22(0.76)$ & $0.05(0.16)$ & $0.06(0.18)$ \\
ESP & $0.04(0.12)$ & $0.04(0.11)$ & $0.05(0.16)$ & $0.05(0.16)$ & $0.11(0.20)$ & $0.15(0.34)$
\end{tabular}

${ }^{a}$ Cutoffs are in au. "All" refers to fitting all cubes with even weight.

every atomic nuclei were discarded. Note that this is somewhat similar to the ESP fitting method; however, in this case there is no long-range cutoff. Furthermore, we have used smaller inner cutoffs than for conventional ESP charge fitting procedures because in this case the fitting functions are continuous (Hermite Gaussians). In the calculations below, the inner cutoffs range from $0.2-1$ au for the linear and $0.76-1.89$ au for the nonlinearleast-squares fits. Finally, the total charge of the molecules was kept constant by using the method of Lagrange multipliers to include the constraint in the fitting procedure.

\section{Results}

Electrostatic and exchange intermolecular interactions were calculated to test the fitting coefficients obtained from the gridded molecular properties. In this section we present the results from these calculations compared to CSOV. In subsection 3.1 we present the results for the investigation of the quality of the fit with respect to the cutoff distance for a set of 10 water dimers. ${ }^{35,36}$ In this case, grids of molecular density, ESP, electric field and "all" grids (evenly weighted) are fitted to the A1 and P1 ABSs. In addition, results are discussed for the same dimers from the nonlinear fit to three and seven s-type Hermite Gaussian functions. Subsequently, in subsection 3.2 we present results for the canonical water dimer, an ammonia-water dimer and a methane-water dimer comparing intermolecular Coulomb interaction energies obtained with the numerical fit and from DF and their dependence with $\lambda$. Finally, in subsection 3.3 we present results for intermolecular exchange interactions for the ten water dimers. In all cases the fitted grids are rectangular unless otherwise stated.

3.1. Intermolecular Coulomb for Water Dimers. In our previous studies we have noted that the ABSs can be placed on different points and need not be restricted to the atomic centers. On this basis, we have fitted two sets of coefficients, one consists of five fitting sites that include the three atomic centers as well as bond midpoints. The second set only comprises ABSs centered on the atoms.

The results obtained for the intermolecular Coulomb interaction are in good agreement with the reference CSOV calculations for both A1 and P1. Tables 1 and 2 show the calculated average and maximum error for all grids fitted to a single molecule with ABSs on the atoms and midpoints. As can be seen, in the cases of the ESP and field fitted coefficients, as well as when fitting all five grids (density, ESP and three components of the field)
TABLE 3: Absolute Average (Maximum) Error in Coulomb Interaction, in $\mathrm{kcal} / \mathrm{mol}$, with Respect to CSOV Using Hermite Gaussians for the 10 Water Dimers When Fitting Grids to A1 on 3 Sites $^{a}$

\begin{tabular}{lccc}
\hline & \multicolumn{3}{c}{ cutoff $(\mathrm{au})$} \\
\cline { 2 - 4 } cube(s) & 1.0 & 0.5 & 0.2 \\
\hline all & $0.05(0.13)$ & $0.12(0.30)$ & $0.16(0.32)$ \\
density & $0.43(1.06)$ & $0.52(1.16)$ & $1.14(2.17)$ \\
ESP & $0.11(0.17)$ & $0.19(0.34)$ & $0.17(0.30)$ \\
field & $0.10(0.27)$ & $0.13(0.28)$ & $0.18(0.37)$
\end{tabular}

${ }^{a}$ Cutoffs are in au. "All" refers to fitting all cubes with even weight.

TABLE 4: Absolute Average (Maximum) Error in Coulomb Interaction, in $\mathrm{kcal} / \mathrm{mol}$, with Respect to CSOV Using Hermite Gaussians for the 10 Water Dimers When Fitting Grids to P1 on 3 Sites $^{a}$

\begin{tabular}{lccc}
\hline & \multicolumn{3}{c}{ cutoff $(\mathrm{au})$} \\
\cline { 2 - 4 } cube(s) & 1.0 & 0.5 & 0.2 \\
\hline all & $0.10(0.18)$ & $0.17(0.39)$ & $0.19(0.48)$ \\
density & $0.53(1.42)$ & $0.45(1.05)$ & $0.55(1.49)$ \\
ESP & $0.12(0.30)$ & $0.11(0.21)$ & $0.12(0.24)$ \\
field & $0.13(0.38)$ & $0.21(0.67)$ & $0.18(0.52)$
\end{tabular}

${ }^{a}$ Cutoffs are in au. "All" refers to fitting all cubes with even weight.

the absolute average error with respect to CSOV is around 0.2 $\mathrm{kcal} / \mathrm{mol}$ for most cases and is relatively stable as the cutoff distance is varied. For the fit of the density grid, the quality of the fit is very poor for both ABSs for large cutoffs and only improves at close range for $\mathrm{P} 1$ and very close range for A1 as would be expected. The Coulomb interactions obtained from the distributed multipoles extracted from the Hermite coefficients are very stable for both A1 and P1 in all cases except for the density grid, with errors around $0.85 \mathrm{kcal} / \mathrm{mol}$ (see Supporting Information).

Tables 3 and 4 present the results for the errors with respect to $\mathrm{CSOV}$ for the grids fitted to a single water molecule with ABSs centered only on the atoms. In this case only three values for the cutoff were explored. As was the case above, the coefficients obtained from the fit of the density grid have a larger error than the other coefficients. However, in the case of the A1 ABS the average error is not as large as before and, in fact, it increases as the cutoff decreases. Note that a more sophisti- 


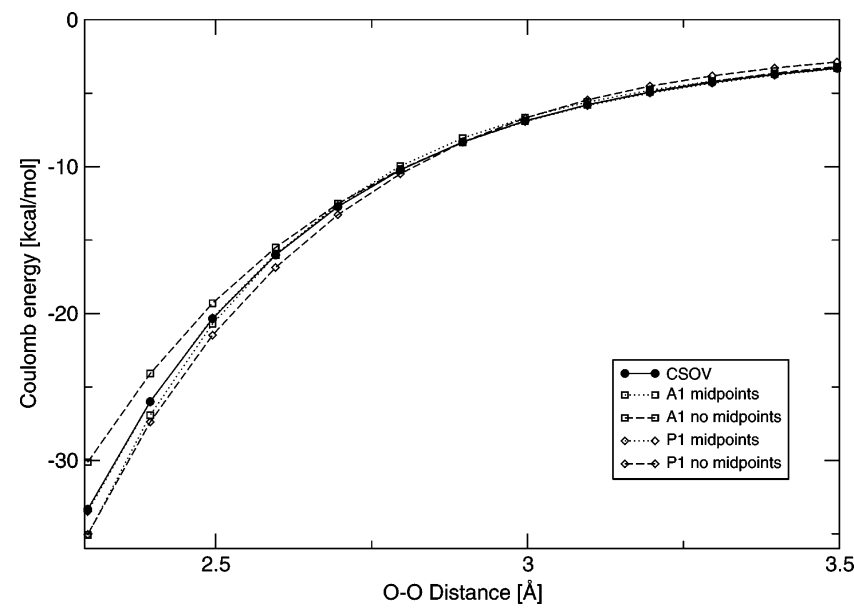

Figure 1. Water dimer (structure 1) Coulomb interaction energies from Hermite coefficients from electrostatic field grids (cutoff $=1.0 \mathrm{au}$ ) for a range of distances.

cated cutoff, for example, based on atom type, would likely improve our results.

Another striking result is that A1 gives better results when no midpoints are employed. This may be due to the fact that in $\mathrm{A} 1$, the basis is more balanced for atoms and the addition of midpoints increases the noise in the fitting procedure. Furthermore, note that when no midpoints are used with $\mathrm{A} 1$, the errors fall well below $0.2 \mathrm{kcal} / \mathrm{mol}$ for every grid except the density one. The multipoles obtained from these coefficients give reasonable results for the intermolecular Coulomb, with average errors between 0.7 and $1.0 \mathrm{kcal} / \mathrm{mol}$ (see Supporting Information).

In addition, the intermolecular Coulomb interaction of the canonical water dimer (dimer 1) was determined using coefficients calculated from 50 randomly oriented water molecules for the A1 ABS using 3 and 5 sites to test the robustness of the fitting procedure. The average interaction energy corresponds to -8.33 and $-8.15 \mathrm{kcal} / \mathrm{mol}$ for the 5 and 3 fitting site models, respectively, with an absolute average (maximum) error of 0.03 $(0.08)$ and $0.31(1.48) \mathrm{kcal} / \mathrm{mol}$.

On the basis of these results, a test was performed to calculate the Coulomb energy on an example 4096 water box in periodic boundary conditions, using an improved version of the extension of the particle mesh Ewald (PME) method. ${ }^{28,37}$ The calculation for this system using the 3 center A1 coefficients fitted from ESP, on a single Xeon CPU at 3.6 GHz takes 2.29 s. In contrast, the same system in sander using TIP3P charges takes $0.2 \mathrm{~s}$ on the same computer. Thus, there is only 1 order of magnitude difference between the grid fitted ABS on atoms and ESP fitted point charges, with the added advantage that the ABSs show much better accuracy (see below).

The neglect of points close to or at the atomic centers may lead to poor intermolecular interactions at close range. To test this, the intermolecular energies were calculated using the canonical water dimer (structure 1 from the 10 dimers), at different distances (see Figure 1). In this case only the coefficients obtained from the electrostatic field with a cutoff of 1.0 au were employed. It is observed that indeed, the error in Coulomb intermolecular interaction increases as the distance decreases; however, the error is still small at hydrogen bonding (H-bonding) distances. In fact, this trend is observed for both the 5 and 3 site models.

For the nonlinear fit, an atom centered model (3 fitting sites) and a model of seven fitting sites were employed. The seven sites for the latter model are located on the atoms (3), bond midpoints (2), and electron lone pairs (2). The fitting coefficients and the exponents for the Hermite Gaussians, were optimized concurrently for a range of inner cutoffs, fitting only to the ESP. In this case only a single s-type Hermite Gaussian was used at each fitting site; in addition, an outer cutoff of $2.8 \AA$ was employed. As can be seen from Table 5, the errors for the atom centered only model show reasonable agreement with an average error of $0.34 \mathrm{kcal} / \mathrm{mol}$ at an inner cutoff of 1.70 au $(0.9 \AA)$, although they exhibit a strong dependence with respect to inner cutoff value.

The seven point model shows better agreement with CSOV, with an average error around $0.20 \mathrm{kcal} / \mathrm{mol}$ for a range of cutoffs (see Table 6). For this model, there is a smaller dependence with respect to the inner cutoff value, compared to the 3 site model above. Note that the nonlinear fit procedure is equivalent to optimizing a molecular ABS, in contrast to the linear fit, where pre-optimized atomic ABSs are employed. Furthermore, the nonlinear fit provides the advantage of a smaller number of Hermite functions, however, there is an added difficulty in the fitting procedure because stable fits can only be found with a limited number of Gaussian functions.

3.2. $\lambda$ Dependence. Tables $7-9$ show the calculated intermolecular Coulomb interaction for the canonical water dimer, a water-ammonia dimer and a water-methane dimer, respectively. In all cases the interactions were calculated with coefficients obtained from DF, as well as fitted from ESP and field grids with a cutoff of $1.0 \mathrm{au}$, for a range of $\lambda$ from $10^{-2}$ to $10^{-8}$. Note that in this case we have also included results for a large ABS (g03), which was generated from the automatic auxiliary basis generator in Gaussian03. ${ }^{38}$

As can be seen from these results, the coefficients obtained from the fit to the numerical grids show less variation with respect to the $\lambda$ used for the fit, as compared with the DF ones. Additionally, as observed above, the coefficients fitted to the A1 ABS with numerical grids show better agreement with CSOV than the DF ones. This is more apparent in the water dimer, where the interaction energy with DF coefficients varies from -9.77 to $-7.55 \mathrm{kcal} / \mathrm{mol}$, compared to -8.00 to -7.83 $\mathrm{kcal} / \mathrm{mol}$ for field grids $(\mathrm{CSOV}=-8.32 \mathrm{kcal} / \mathrm{mol})$. In the case of ESP fit, the coefficients show stability between $10^{-2}$ and $10^{-7}$ and break down when $\lambda=10^{-8}$.

In the case of the multipoles extracted from the Hermite coefficients, the same trend is observed between the analytic (DF) and numerical (grids) fit, albeit to a lesser extent. For example, in the case of the water dimer with A1 ABS the calculated intermolecular Coulomb interaction with DF coefficients varies around $1 \mathrm{kcal} / \mathrm{mol}$ when the $\lambda$ is varied, with an average value of $-6.70 \mathrm{kcal} / \mathrm{mol}$ for the interaction. In contrast, the variation for the coefficients fitted from both the ESP and field grids vary less than $0.1 \mathrm{kcal} / \mathrm{mol}$ along the entire $\lambda$ range, with an average interaction value of -6.60 and $-6.75 \mathrm{kcal} /$ mol for ESP and field, respectively.

On the basis of the results above, it was decided to test the intramolecular interactions to determine the source of the dependence with respect to $\lambda$. Because, for all practical purposes, the difference between the DF and grid fits is the neglect of the core contributions for the latter, it is possible to compare intramolecular energies calculated with coefficients from both procedures. From these results it is observed that the error in the DF fit does indeed come from the attempt of the ABS to fit the core, and this error is reduced by increasing the quality of the ABS, e.g., from A1 to P1.

For example, for A1, the nuclear-electron intramolecular energies from $a b$ initio, DF and field grids fit with 1.0 au cutoff 
TABLE 5: Absolute Average (Maximum) Error in Coulomb Interaction with Respect to CSOV in kcal/mol, for Nonlinear Hermite Fit When Fitting ESP Grids to s-Type Hermites Only, 3 Site Model $^{a}$

\begin{tabular}{ccccccc}
\hline \multicolumn{7}{c}{ cutoff $(\mathrm{au})$} \\
\cline { 2 - 5 } 1.89 & 1.70 & 1.51 & 1.32 & 1.13 & 0.94 & 0.76 \\
\hline $0.36(0.77)$ & $0.34(0.70)$ & $0.60(1.01)$ & $0.93(1.58)$ & $1.27(2.15)$ & $1.61(2.69)$ & $1.89(3.15)$
\end{tabular}

${ }^{a}$ Cutoffs are in au. Only one s-type Hermite function is employed on each site.

TABLE 6: Absolute Average (Maximum) Error in Coulomb Interaction with Respect to CSOV in kcal/mol, for Nonlinear Hermite Fit When Fitting ESP Grids to s-Type Hermites Only, 7 Site Model $^{a}$

\begin{tabular}{ccccccc}
\hline \multicolumn{7}{c}{ cutoff $(\mathrm{au})$} \\
\hline 1.89 & 1.70 & 1.51 & 1.32 & 1.13 & 0.94 & 0.75 \\
\hline $0.27(0.71)$ & $0.17(0.35)$ & $0.22(0.42)$ & $0.34(0.64)$ & $0.38(0.82)$ & $0.31(0.88)$ & $0.25(0.88)$
\end{tabular}

${ }^{a}$ Cutoffs are in au. Only one s-type Hermite function is employed on each site.

TABLE 7: Water-Water Coulomb Interaction, Structure $1(\mathrm{CSOV}=-8.32)$ for Analytic Density Fitting (DF), and Numerical Fitting for ESP and Field Grids ${ }^{a}$

\begin{tabular}{|c|c|c|c|c|c|c|c|c|c|}
\hline \multirow[b]{2}{*}{$\lambda$} & \multicolumn{3}{|c|}{ A1 } & \multicolumn{3}{|c|}{ P1 } & \multicolumn{3}{|c|}{ g03 } \\
\hline & full DF & ESP & FLD & full DF & ESP & FLD & full $\mathrm{DF}$ & ESP & FLD \\
\hline $10^{-2}$ & $-9.77(-5.91)$ & $-8.12(-6.60)$ & $-8.00(-6.75)$ & $-6.84(-6.27)$ & $-8.41(-6.33)$ & $-8.41(-6.33)$ & $-9.46(-5.77)$ & $-8.92(-6.61)$ & $-8.30(-6.53)$ \\
\hline $10^{-3}$ & $-9.15(-6.61)$ & $-8.34(-6.59)$ & $-8.04(-6.80)$ & $-8.08(-6.44)$ & $-8.40(-6.42)$ & $-8.40(-6.42)$ & $-8.90(-6.04)$ & $-8.50(-6.60)$ & $-8.20(-6.54)$ \\
\hline $10^{-4}$ & $-7.90(-6.73)$ & $-8.39(-6.60)$ & $-8.05(-6.81)$ & $-8.59(-6.22)$ & $-8.35(-6.50)$ & $-8.35(-6.50)$ & $-8.63(-6.30)$ & $-8.21(-6.51)$ & $-8.24(-6.54)$ \\
\hline $10^{-5}$ & $-7.85(-6.69)$ & $-8.38(-6.61)$ & $-8.02(-6.78)$ & $-8.44(-6.21)$ & $-8.33(-6.51)$ & $-8.33(-6.51)$ & $-8.38(-6.47)$ & $-8.24(-6.52)$ & $-8.26(-6.54)$ \\
\hline $10^{-6}$ & $-7.94(-6.70)$ & $-8.33(-6.63)$ & $-7.90(-6.75)$ & $-8.33(-6.48)$ & $-8.29(-6.52)$ & $-8.29(-6.52)$ & $-8.30(-6.54)$ & $-8.25(-6.51)$ & $-8.27(-6.51)$ \\
\hline $10^{-7}$ & $-7.96(-6.71)$ & $-7.87(-6.63)$ & $-7.86(-6.74)$ & $-8.32(-6.59)$ & $-8.21(-6.52)$ & $-8.24(-6.53)$ & $-8.31(-6.55)$ & $-8.47(-6.57)$ & $-8.27(-6.48)$ \\
\hline $10^{-8}$ & $-7.95(-6.72)$ & $-5.63(-6.62)$ & $-7.83(-6.75)$ & $-8.32(-6.59)$ & $-8.20(-6.53)$ & $-8.18(-6.54)$ & $-8.32(-6.55)$ & $-8.14(-6.50)$ & $-9.12(-6.48)$ \\
\hline
\end{tabular}

TABLE 8: Ammonia-Water Coulomb Interaction for DF, and Numerical Fitting for ESP and Field Grids $(\mathrm{CSOV}=-1.17)^{a}$

\begin{tabular}{|c|c|c|c|c|c|c|c|c|c|}
\hline \multirow[b]{2}{*}{$\lambda$} & \multicolumn{3}{|c|}{ A1 } & \multicolumn{3}{|c|}{$\mathrm{P} 1$} & \multicolumn{3}{|c|}{$\mathrm{g} 03$} \\
\hline & full DF & ESP & FLD & full DF & ESP & FLD & full DF & ESP & FLD \\
\hline $10^{-2}$ & $-1.51(-1.15)$ & $1.26(-0.90)$ & $-1.18(-0.91)$ & $-1.04(-0.89)$ & $-1.22(-0.86)$ & $-1.23(-0.84)$ & $-1.16(-0.90)$ & $-1.18(-0.84)$ & $-1.17(-0.89)$ \\
\hline $10^{-3}$ & $-1.39(-1.06)$ & $-1.20(-0.90)$ & $-1.12(-0.92)$ & $-1.19(-0.94)$ & $-1.20(-0.88)$ & $-1.20(-0$. & $-1.13(-0.89)$ & $-1.17(-0.88)$ & $-1.16(-0.89)$ \\
\hline $10^{-4}$ & $-1.23(-1.01)$ & $-1.18(-0.90)$ & $-1.11(-0.93)$ & $-1.18(-0.93)$ & $-1.20(-0.90)$ & $-1.22(-0$ & $-1.17(-0.89)$ & $-1.17(-0.90)$ & $-1.16(-0.89)$ \\
\hline $10^{-}$ & -1.1 & -1.18 & $-1.12(-$ & $-1.24(-0$. & -1.19( & -1.21( & $-1.16(-0.90)$ & -1.16 & -1.16 \\
\hline $10^{-6}$ & $-1.12(-0.93$ & $-1.19(-0.90)$ & $-1.17(-0.92)$ & $-1.27(-0.89)$ & $-1.20(-0.91)$ & $-1.20(-0.89)$ & $-1.16(-0.90)$ & $-1.15(-0.89)$ & $-1.16(-0.89)$ \\
\hline $10^{-7}$ & $-1.11(-0.93)$ & $-1.22(-0.90)$ & $-1.19(-0.93)$ & $-1.29(-0.89)$ & $-1.19(-0.90)$ & $-1.21(-0.89)$ & $-1.16(-0.90)$ & $-1.01(-0.82)$ & $-1.16(-0.88)$ \\
\hline $10^{-8}$ & $-1.12(-0.93)$ & $-1.18(-0.90)$ & $-1.19(-0.93)$ & $-1.30(-0.89)$ & $-1.19(-0.91)$ & $-1.22(-0.89)$ & $-1.16(-0.90)$ & $-1.17(-0.81)$ & $-1.16(-0.87)$ \\
\hline
\end{tabular}

${ }^{a}$ Numbers in parentheses correspond to multipoles ( $\left.\mathrm{kcal} / \mathrm{mol}\right)$. All interactions were calculated with corresponding coefficients, e.g., A1 ESP coefficients for both $\mathrm{H}_{2} \mathrm{O}$ and $\mathrm{NH}_{3}$, or P1 FLD for both $\mathrm{H}_{2} \mathrm{O}$ and $\mathrm{NH}_{3}$.

TABLE 9: Methane-Water Coulomb Interaction for DF, and Numerical Fitting for ESP and Field Grids $(\mathrm{CSOV}=-0.42)^{a}$

\begin{tabular}{|c|c|c|c|c|c|c|c|c|c|}
\hline \multirow[b]{2}{*}{$\lambda$} & \multicolumn{3}{|c|}{ A1 } & \multicolumn{3}{|c|}{ P1 } & \multicolumn{3}{|c|}{ g03 } \\
\hline & full DF & ESP & FLD & full DF & ESP & FLD & full DF & ESP & FLD \\
\hline $10^{-2}$ & $-0.56(0.17)$ & $-0.36(0.09$ & $25(0112)$ & $0.25(0.27)$ & $-0.47(0.10)$ & $0.45(0.09)$ & $-0.43(0.09)$ & $-0.39(0.14)$ & $-0.41(0.10)$ \\
\hline $10^{-3}$ & $-0.54(0.06)$ & $-0.38(0.09)$ & $-0.37(0.11)$ & $-0.45(0.12)$ & $-0.45(0.07)$ & $-0.38(0.08)$ & $-0.41(0.10)$ & $-0.42(0.11)$ & $-0.41(0.10)$ \\
\hline $10^{-4}$ & $-0.22(0.09)$ & $-0.40(0.09)$ & $-0.41(0.09)$ & $-0.52(0.05)$ & $-0.44(0.09)$ & $-0.38(0.08)$ & $-0.41(0.10)$ & $-0.42(0.10)$ & $-0.42(0.10)$ \\
\hline $10^{-5}$ & $-0.18(0.10)$ & $-0.41(0.09)$ & $-0.40(0.08)$ & $-0.55(0.08)$ & $-0.44(0.09)$ & $-0.42(0.11)$ & $-0.41(0.10)$ & $-0.43(0.09)$ & $-0.43(0.10)$ \\
\hline $10^{-6}$ & $-0.31(0.10)$ & $-0.42(0.09)$ & $-0.41(0.09)$ & $-0.53(0.09)$ & $-0.44(0.11)$ & $-0.45(0.11)$ & $-0.41(0.10)$ & $-0.44(0.09)$ & $-0.42(0.10)$ \\
\hline $10^{-7}$ & $-0.41(0.10)$ & $-0.44(0.09)$ & $-0.42(0.10)$ & $-0.50(0.10)$ & $-0.44(0.11)$ & $-0.46(0.09)$ & $-0.42(0.10)$ & $-0.43(0.10)$ & $-0.42(0.10)$ \\
\hline $10^{-8}$ & $-0.43(0.10)$ & $-0.41(0.10)$ & $-0.42(0.10)$ & $-0.48(0.10)$ & $-0.44(0.11)$ & $-0.47(0.08)$ & $-0.42(0.10)$ & $-0.45(0.06)$ & $-0.42(0.11)$ \\
\hline
\end{tabular}

${ }^{a}$ Numbers in parentheses correspond to multipoles $(\mathrm{kcal} / \mathrm{mol})$. All interactions were calculated with corresponding coefficients, e.g., A1 ESP coefficients for both $\mathrm{H}_{2} \mathrm{O}$ and $\mathrm{CH}_{4}$, or P1 FLD for both $\mathrm{H}_{2} \mathrm{O}$ and $\mathrm{CH}_{4}$.

are $-198.886,-198.944$, and 248.572 au, respectively. As can be seen, the DF fitted density is in agreement to the $a b$ initio one; however, there is an error of 0.06 au due to the ABS not fitting the core contribution correctly because of its small size. On the other hand, the intramolecular energy calculated with the coefficients fitted from the field grids does not agree with the $a b$ initio one, and it even has the wrong sign. This is due to the neglect of the grid points within 1 au of the atomic cores. However, once the ABS does not have to fit the core contributions, it can perform better fitting the valence, which is the part involved in inter-molecular interactions.
Furthermore, this may also be explained by calculating the electrostatic field components with $a b$ initio and DF. As noted in our previous study, ${ }^{23}$ the molecular properties calculated with $\mathrm{DF}$ are in good agreement with their respective $a b$ initio values at points not close to the cores. For instance, for a water molecule in the standard geometry used in this study, the three components of the field at the $\mathrm{O}$ atom position are -0.126784 , 0.0, 0.0 and $-0.33168,0.0,0.0$ au for ab initio and A1 fitted with DF, respectively. In contrast, the values at 0.5 and $2.0 \AA$ are 3.958363, 0.0, $0.0(3.970649,0.0,0.0)$ and 0.020407, 0.0, $0.0(0.020234,0.0,0.0)$ au for $a b$ initio (A1), respectively. As 
TABLE 10: Counterpoise Corrected Total Energies, CSOV Coulomb and CHELPG Coulomb Intermolecular Interactions for the 10 Water Dimers, in $\mathrm{kcal} / \mathrm{mol}$

\begin{tabular}{lccccccccccc}
\hline & \multicolumn{7}{c}{ dimer } \\
\cline { 2 - 9 } & 1 & 2 & 3 & 4 & 5 & 6 & 7 & 8 & 9 & 10 \\
\hline total ab initio & -5.62 & -4.94 & -4.91 & -4.20 & -3.80 & -3.73 & -3.07 & -1.20 & -3.07 & -2.33 \\
CHELPG Coulomb & -5.31 & -4.90 & -5.08 & -4.66 & -4.33 & -4.51 & -3.57 & -0.99 & -4.07 & -3.22 \\
CSOV Coulomb & -8.32 & -7.02 & -7.00 & -6.23 & -5.59 & -5.43 & -4.35 & -1.31 & -4.79 & -3.09
\end{tabular}

can be seen, the error decreases dramatically as we move away from the core.

To put the above results in context, the electric field contributions at the same points using CHELPG $^{9}$ fitted charges have been calculated in the same geometry. We have used CHELPG charges because these are obtained from a similar procedure. In this case, CHELPG (charges from electrostatic potentials using a grid based method) charges are fitted to reproduce the molecular electrostatic potential at a number of points around the molecule. The calculated field components are $-0.136775,0.0,0.0 ;-0.872098,0.0,0.0$ and 0.018767 , $0.0,0.0$ at the $\mathrm{O}$ core, 0.5 and $2.0 \AA$, respectively. Notably, the CHELPG charges give better agreement at the core than DF. The error at $0.5 \AA$ is very large, as is to be expected because this point is outside the cutoff values. However, note that even at $2.0 \AA$, the error for the permanent field is 1 order of magnitude larger for CHELPG charges compared with DF (1.0 vs $0.1 \mathrm{kcal} /$ mol).

Moreover, it is interesting to point out that the intermolecular Coulomb interaction calculated with these charges shows an rms deviation of $1.58 \mathrm{kcal} / \mathrm{mol}$ with respect to CSOV (see Table 10). Compare this result with the average errors of the grid fitting (linear and nonlinear), which range between 0.46 and $0.19 \mathrm{kcal} / \mathrm{mol}$ for the nonlinear fit and are well below $0.2 \mathrm{kcal} /$ mol for the linear fit. However, note that the intermolecular Coulomb energies calculated with the CHELPG charges are relatively close to the total ab initio basis set superposition error (BSSE) corrected intermolecular interaction energies for several of these water dimers. However, there are some cases where the total $a b$ initio energies differ by around $1 \mathrm{kcal} / \mathrm{mol}$ compared to the Coulomb energies from CSOV depending on the dimer orientation.

As can be seen, the total energies are very close to the Coulomb energy calculated with ESP fitted charges, as previously noted by Dunitz and Gavezzotti. ${ }^{39}$ Therefore, for a conventional nonpolarizable pairwise force field, the Van der Waals term only has to correct a very small part of the total energy, relying on error cancellations. However, if polarization is to be included, the error will be 2-fold. First, the use of a classical polarization model will produce errors because the permanent fields calculated with the ESP fitted charges are incorrect, as shown above. Additionally, it is to be expected that the Coulomb interaction from CHELPG charges for a random orientation will not be close to the total energy, thus the Van der Waals term will most likely fail in canceling this error.

3.3. Intermolecular Exchange for Water Dimers. Finally, the coefficients obtained from the grid fit were tested for the calculation of the intermolecular exchange interactions for the ten water dimers. Here, only two values of $\lambda$ were investigated, 1.0 and $0.5 \mathrm{au}$. In the case of the density grid fitted coefficients, the $\lambda$ values considered were 0.5 and 0.2 instead because these were the values that gave reasonable Coulomb interactions energies. For all results shown in Tables 11 and 12 the $K$ values for the overlap model were obtained from a linear regression to all ten water dimers (see Supporting Information).
TABLE 11: Absolute Average (Maximum) Error in Exchange Interaction, in $\mathrm{kcal} / \mathrm{mol}$, with Respect to CSOV Using Hermite Gaussians for the 10 Water Dimers When Fitting Grids to A1 on 5 Sites $^{a}$

\begin{tabular}{lcc}
\hline & \multicolumn{2}{c}{ cutoff $(\mathrm{au})$} \\
\cline { 2 - 3 } cube $(\mathrm{s})$ & 1.0 & 0.5 \\
\hline all & $0.33(0.69)$ & $0.18(0.49)$ \\
density $^{b}$ & $0.15(0.42)$ & $0.76(1.16)$ \\
ESP & $0.15(0.41)$ & $0.27(0.54)$ \\
field $^{c}$ & $0.23(0.61)$ & $0.31(0.82)$
\end{tabular}

${ }^{a}$ Cutoffs are in au. "All" refers to fitting all cubes with even weight. ${ }^{b}$ Values are for cutoffs at 0.5 and 0.2 instead of 1.0 and 0.5 , respectively. ${ }^{c}$ Value for cutoff of 1.0 corresponds to 0.8 instead (see text).

TABLE 12: Absolute Average (Maximum) Error in Exchange Interaction with Respect to CSOV, in $\mathrm{kcal} / \mathrm{mol}$, Using Hermite Gaussians for the 10 Water Dimers When Fitting Grids to P1 on 5 Sites $^{a}$

\begin{tabular}{rcc}
\hline & \multicolumn{2}{c}{ cutoff $(\mathrm{au})$} \\
\cline { 2 - 3 } cube(s) & 1.0 & 0.5 \\
\hline all & $0.14(0.34)$ & $0.20(0.45)$ \\
density $^{b}$ & $0.48(1.23)$ & $0.39(1.23)$ \\
ESP & $0.15(0.38)$ & $0.20(0.45)$ \\
field & $0.16(0.34)$ & $0.20(0.45)$
\end{tabular}

${ }^{a}$ Cutoffs are in au. "All" refers to fitting all cubes with even weight. ${ }^{b}$ Values are for cutoffs at 0.5 and 0.2 instead of 1.0 and 0.5 , respectively (see text).

As can be seen from Tables 11 and 12, the coefficients obtained from fitting ESP, field, and all cubes evenly weighted show good agreement with CSOV, especially in the case of P1 where the errors are all at or below $0.2 \mathrm{kcal} / \mathrm{mol}$. In the case of the density grid, it is interesting to point out that in the case of A1, the error increases as $\lambda$ decreases opposite to the Coulomb results. This may be due to the fact that, as explained in the previous subsection, the ABS may be trying to fit the core, whereas for the overlap model, it is the valence contributions that are important for the determination of the intermolecular exchange interaction. ${ }^{33}$ These results also justify empirical models such as the sum of interactions between fragments $a b$ initio (SIBFA), which includes an exchange-repulsion term based on valence-valence interactions only. ${ }^{22,40}$

In the case of the nonlinear fit, the coefficients corresponding to the best average Coulomb interaction, cutoff of 1.89 and 0.75 au for 3 and 7 site fits, respectively, were used to calculate the exchange interaction. For the 3 site model, an absolute average error of $0.37 \mathrm{kcal} / \mathrm{mol}$ was obtained, with a maximum error of $1.0 \mathrm{kcal} / \mathrm{mol}$. In the case of the 7 site model, the absolute average and maximum errors are 0.4 and $1.26 \mathrm{kcal} / \mathrm{mol}$, respectively.

Finally, the energy scan performed in subsection 3.1 was explored for the exchange interaction. Again, only the coefficients obtained from the electrostatic field grids with a cutoff of $1.0 \mathrm{au}$ were employed. As can be seen from Figure 2, the trend observed for the Coulomb interactions is followed for exchange. That is, the error is small at medium and long range and increases as the distance between the monomers decreases. Another possible source of error is the calculation of the $K$ 


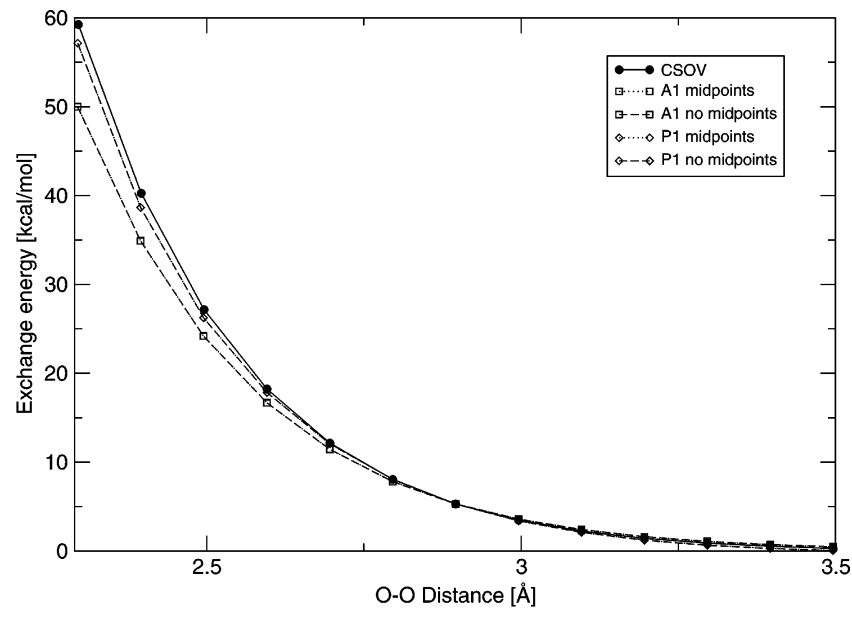

Figure 2. Water dimer (structure 1) exchange interaction energies from Hermite coefficients from electrostatic field grids (cutoff $=1.0 \mathrm{au}$ ) for a range of distances.

parameter for the overlap model; in this case, the $K$ was calculated from the fit to one single point on the curve (equilibrium distance of the water dimer).

\section{Conclusions}

A method has been developed for the determination of fitting coefficients for ABSs based on the fitting of gridded molecular properties, as well as a nonlinear fit that allows the concurrent optimization of coefficients and exponents in the basis set. These coefficients may be used for the determination of intermolecular Coulomb and exchange interaction energies. The molecular properties used in this study include density, ESP, electrostatic field, and a combination of all three. In all cases it was shown that a robust fit can be achieved by neglecting the grid points close to the atomic cores. This procedure also shows that, by neglecting the core contributions, the coefficients are less dependent on the parameters of the fitting procedure. By fitting to grids, it is possible to use less fitting sites, which leads to increased computational efficiency. This is demonstrated by the fact that a single energy calculation for a 4096 water box takes roughly 1 order of magnitude more time for the fitted Hermites than for conventional point charges. In addition, it was shown that the coefficients obtained from the numerical fitting can be also used to determine the exchange interaction by means of the overlap model. Alternative grids and higher order Hermites for the nonlinear fit are currently under investigation.

Acknowledgment. This research was supported in part by the intramural research program of the NIH and NIEHS. Computing time from the advanced biomedical computing center NCI-FCRDC, as well as helpful comments by the referees, are gratefully acknowledged.

Supporting Information Available: Absolute average and maximum errors for the 10 water dimers for 5 and 3 site fits, fitting parameters and error plots for the nonlinear-least-squares procedure, optimized coefficients and $\exp$ [onents for the water models, error plots for the exchange contributions and $K$ values for the exchange interactions are presented. This material is available free of charge via the Internet at http://pubs.acs.org.

\section{References and Notes}

(1) Case, D. A.; Cheatham, T. E., III; Darden, T. A.; Gohlke, H.; Luo, R.; Merz, K. M., Jr.; Onufirev, A.; Simmerling, C.; Wang, B.; Woods, R. J. J. Comp. Chem. 2005, 26, 1668-1688. 3616.

(2) MacKerell, A. D., Jr.; et al. J. Phys. Chem. B 1998, 102, 3586-

(3) van der Spoel, D.; Lindahl, E.; Hess, B.; Groenhoff, G.; Mark, A. E.; Berensen, H. J. C. J. Comput. Chem. 2005, 26, 1701-1718.

(4) Christen, M.; Hünenberger, P. H.; Bakowies, D.; Baron, R.; Brúgl, R.; Geerke, D. P.; Heinz, T. N.; Kastenholz, M. A.; Kräutler, V.; Oostenbrink, C.; Peter, C.; Trzesniak, D.; van Gunsteren, W. F. J. Comput. Chem. 2005, 26, 1719-1751.

(5) Jorgensen, W. L.; Tirado-Rives, J. J. Comput. Chem. 2005, 26, $1669-1700$

(6) Leach, A. R. Molecular modelling; principles and applications, 2nd ed.; Prentice Hall: Harlow, U.K., 2001.

(7) Mulliken, R. S. J. Chem. Phys. 1955, 23, 1833-1840.

(8) Bader, R. F. W. Atoms in molecules: a quantum theory; Clarendon Press: Oxford, U.K., 1990 373.

(9) Breneman, C. M.; Wiberg, K. B. J. Comput. Chem. 1990, 11, 361-

(10) Bayly, C. I.; Cieplak, P.; Cornell, W. D.; Kollman, P. A. J. Phys. Chem. 1993, 97, 10269-10280.

(11) Hu, H.; Lu, Z.; Yang, W. J. Chem. Theo. Comput. 2007, 3, 10041013.

(12) Stone, A. J. The theory of intermolecular forces; Oxford University Press: Oxford, U.K., 2000.

(13) Stone, A. J. Chem. Phys. Lett. 1981, 83, 233-239.

(14) Vigné-Maeder, F.; Claverie, P. J. Chem. Phys. 1988, 88, 49344948.

(15) Ángyán, J. G.; Chipot, C.; Dehez, F.; Hätig, C.; Jansen, G.; Millot, C. OPEP, A tool for the optimal partitioning of electric properties version 1.0- $\beta$. Equipe de chimie and biochimie théoretiques, Unité mixte de recherche CNRS/UHP No 7565, Université Henri Poincaré, B.P. 239, 54506 Vandoeuvre-lés-Nancy, Cedex, France, 2005.

(16) Sagui, C.; Pedersen, L. G.; Darden, T. A. J. Chem. Phys. 2004, $120,73-87$.

(17) Gresh, N.; Claverie, P.; Pullman, A. Int. J. Quantum Chem. 1979, 253, Symp 11.

(18) Gresh, N. J. Comput. Chem. 1995, 16, 856-882.

(19) Ren, P.; Ponder, J. W. J. Phys. Chem. B 2003, 107, 5933-5947.

(20) Freitag, M. A.; Gordon, M. S.; Jensen, J. H.; Stevens, W. J. J. Chem. Phys. 2000, 112, 7300-7306.

(21) Piquemal, J.-P.; Gresh, N.; Giessner-Prettre, C. J. Phys. Chem. A 2003, 107, 10353-10359.

(22) Piquemal, J.-P.; Chevreau, H.; Gresh, N. J. Chem. Theo. Comput. 2007, 3, 824-837.

(23) Cisneros, G. A.; Piquemal, J.-P.; Darden, T. A. J. Chem. Phys. 2005, 123, 044109 .

(24) Boys, S. F.; Shavit, I. A Fundamental Calculation of the Energy Surface for the System of Three Hydrogen Atoms; AD212985; NTIS: Springfield, VA, 1959

(25) Dunlap, B. I.; Connolly, J. W. D.; Sabin, J. R. J. Chem. Phys. 1979 $71,4993-4999$.

(26) Köster, A. M.; Calaminici, P.; Gómez, Z.; Reveles, U. Density functional theory calculation of transition metal clusters. Reviews of Modern Quantum Chemistry, A Celebration of the Contribution of Robert G. Parr; World Scientific: Singapore, 2002

(27) Piquemal, J.-P.; Cisneros, G. A.; Reinhardt, P.; Gresh, N.; Darden, T. A. J. Chem. Phys. 2006, 124, 104101.

(28) Cisneros, G. A.; Piquemal, J.-P.; Darden, T. A. J. Chem. Phys. 2006, 125, 184101.

(29) Elking, D.; Darden, T. A.; Woods, R. J. J. Comput. Chem. 2007, $28,1261-1274$

(30) Bagus, P. S.; Illas, F. J. Chem. Phys. 1992, 96, 8962-8960.

(31) Piquemal, J.-P.; Marquez, A.; Parisel, O.; Giessner-Prettre, C. J. Comput. Chem. 2005, 26, 1052-1062.

(32) Press, W. H.; Teukolsky, S. A.; Vetterling, W. T.; Flannery, B. P. Numerical recipes in fortran77; the art of scientific computing, 2nd ed.; Cambridge University Press: New York, 1992.

(33) Wheatley, R. J.; Price, S. L. Mol. Phys. 1990, 69, 507-533.

(34) Frisch, M. J.; Trucks, G. W.; Schlegel, H. B.; Scuseria, G. E.; Robb, M. A.; Cheeseman, J. R.; Zakrzewski, V. G.; Montgomery, J. A.; Stratmann, R. E.; Burant, J. C.; Dapprich, S.; Millam, J. M.; Daniels, A. D.; Kudin, K. N.; Strain, M. C.; Farkas, O.; Tomasi, J.; Barone, V.; Cossi, M.; Cammi, R.; Mennucci, B.; Pomelli, C.; Adamo, C.; Clifford, S.; Ochterski, J.; Petersson, G. A.; Ayala, P. Y.; Cui, Q.; Morokuma, K.; Malick, D. K., Rabuck, A. D.; Raghavachari, K.; Foresman, J. B.; Cioslowski, J.; Ortiz, J. V.; Stefanov, B. B.; Liu, G., Liashenko, A., Piskorz, P.; Komaromi, I.; Gomperts, R.; Martin, R. L.; Fox, D. J.; Keith, T.; Al-Laham, M. A.; Peng, C. Y.; Nanayakkara, A.; Gonzalez, C.; Challacombe, M.; Gill, P. M. W.; Johnson, B.; Chen, W.; Wong, M. W.; Andres, J. L.; Head-Gordon, M.; Replogle, E. S. and Pople, J. A. Gaussian 98, revision A.8; Gaussian, Inc.: Pittsburgh, PA, 1998

(35) Tschumper, G. S.; Leininger, M. L.; Hoffman, B. C.; Valeev, E. F.; Schaffer, H. F., III; Quack, M. J. Chem. Phys. 2002, 116, 690-701. 
(36) van Duijneveldt-van, de Rijdt, J. G. C. M.; Mooij, W. T. M.; Duijneveldt, F. B. Phys. Chem. Chem. Phys. 2003, 5, 1169-1180.

(37) Darden, T. A. Dual bases in crystallographic computing. In International Tables of Chrystallography; Kluwer Academic Publishers: Dordrecht, The Netherlands, 2008; Vol. B, 3rd ed., in press.

(38) Frisch, M. J.; Trucks, G. W.; Schlegel, H. B.; Scuseria, G. E.; Robb, M. A.; Cheeseman, J. R.; Zakrzewski, V. G.; Montgomery, J. A.; Stratmann, R. E.; Burant, J. C.; Dapprich, S.; Millam, J. M.; Daniels, A. D.; Kudin, K. N.; Strain, M. C.; Farkas, O.; Tomasi, J.; Barone, V.; Cossi, M.; Cammi, R.; Mennucci, B.; Pomelli, C.; Adamo, C.; Clifford, S.; Ochterski, J.; Petersson, G. A.; Ayala, P. Y.; Cui, Q.; Morokuma, K.; Malick, D. K.;
Rabuck, A. D.; Raghavachari, K.; Foresman, J. B.; Cioslowski, J.; Ortiz, J. V.; Stefanov, B. B.; Liu, G.; Liashenko, A.; Piskorz, P.; Komaromi, I.; Gomperts, R.; Martin, R. L.; Fox, D. J.; Keith, T.; Al-Laham, M. A.; Peng, C. Y.; Nanayakkara, A.; Gonzalez, C.; Challacombe, M.; Gill, P. M. W.; Johnson, B.; Chen, W.; Wong, M. W.; Andres, J. L.; Head-Gordon, M.; Replogle, E. S. and Pople, J. A. Gaussian 03; Gaussian, Inc.: Pittsburgh PA, 2003.

(39) Dunitz, J. D.; Gavezzotti, A. Angew. Chem., Int. Ed. 2005, 44, $1766-1787$

(40) Gresh, N.; Cisneros, G. A.; Darden, T. A.; Piquemal, J.-P. J. Chem. Theo. Comp., DOI: 10.1021/ct700134r. 